\title{
Selection of Strategies for Commercialized Operation of Stadium
}

\author{
Yanjin Zhang \\ Guangzhou Vocational Institute of Sports, Guangzhou, 510560, China
}

\begin{abstract}
Keywords: stadium; commercialized operation; strategy
\end{abstract}
\begin{abstract}
In the process of development of sports industry in China, stadium is one of the most important material bases, playing the important role of holding various sports events and national fitness. At present, how to reasonably use the sports stadium, commercialize them and provide various cultural and sports activities for people has become a problem urgently to be solved. Based on the above background, in this paper, the author briefly analyzes the commercialized operation overview of stadium, makes some simple discussion aimed at some problems exposed in the commercialized operation of stadium and provides the corresponding commercialized operation strategies on this basis, hoping to provide some references for the smooth commercialized operation of stadium.
\end{abstract}

\section{Introduction}

Sports stadium is one of the most important material bases in the process of development of sports industry, playing the important role of holding various sports events and national fitness. In recent years, with the high-speed development of market economy, the stadiums in China have gradually changed the original survival state and cater to the development trend of the new times by taking advantage of market means. Although many stadiums have reformed their financial management system and other regulations and systems in order to adapt to the social requirements, the commercialized management of many medium and large stadiums still stay at the trend of extensive management, and the phenomenon of no separation between ownership and operation right in the management of stadium, directly causing that the operation right of stadium erodes the ownership, and even the ownership interferes with the operation right to the extent that the sound development of stadium is seriously influenced. Based on this, in this paper, the author briefly analyzes the problems existing in the commercialized operation of sports stadium and the strategies to solve the problems.

\section{Analysis on commercialization of stadium}

Before the reform and opening up, the stadiums in China were state-owned, mainly used for the competition of state teams and the sports activities of schools, enterprises and institutions, but after the reform and opening up, especially after the confirmation of the market economy system, sports industry has been commercialized gradually, and in nature, great changes have happened to the sports stadium. Since the 21st century, people's living standards have been increased, and health investment has occupied an important position in family investment, directly increasing the sports activities. Stadium is the main place for people to exercise and at present, many stadium managers take this opportunity to fully play the fitness function of stadiums, obtaining the corresponding efficiency.

Stadiums have very high utilization rate, not only can they be used to hold various sports events, but also are used to hold various large entertainment activities, with diversified operation modes. Sports stadiums can bring huge economic and social benefits in the process of undertaking some sports events. It is worth noting that while undertaking the sports events, they can also publicize them to expand the social influence and lay a market for the development of commercialization. In recent years, China has held Olympic Games, Paralympic Games and other large sports activities, brining great economic benefit to China.

Holding entertainment activities in stadiums is also an approach for the commercialization of 
stadiums. With the continuous increasing of people's spiritual culture, there have been more and more various large entertainment activities, the audience capacity of stadiums is also larger and larger, and together with their high popularity, many entertainment activities hosts first select stadiums as the holding place. In recent years, the entertainment activities dominated by pop music performance has been transferred to the stadiums, and this is a new economic source for the commercialization of stadiums. In addition, some exhibitions related with sports have also been held in the stadiums successively, making the commercialization of stadium obtain new economic guarantee.

\section{Problems existing in commercialized operation of stadiums}

(I) Lack of guarantee in policy and regulations

At present, the state and government are constructing the regulations on and regulating the management of stadiums according to the law of commercialized operation, the current stadiums in these aspects still have certain problems, like serious hysteresis nature, directly causing lack of regulated management mechanism and unified coordination mechanism and poor commercialized operation environment. The construction of stadiums in China needs to be approved by the government, since the 21st century, people have increased their awareness to the commercialized operation of stadiums, but there still exists certain hysteresis nature in laws, regulations and policy. Currently, the policies and regulations on the commercialization of stadiums have not been so imperfect, and there is a lack of regulated management, and besides, there is too much government interference in the commercialization of stadiums, restricting the road to commercialization of stadiums.

At the same time, in the process of commercialization of stadiums, for benefit, various parties manage their operation, and there is no corresponding coordination and regulation to these groups, because of these problems, the following problems have occurred to the stadiums in the process of commercialization: first, the lack of laws directly causes the phenomenon of multiple management, and the situation is very serious, directly causing low commercialization efficiency and economic benefit; second, due to benefit driving among various departments, and together with the unsmooth information exchange between departments, there is a direct disconnection between links in commercialization process, finally causing high cost of commercialized operation.

(II) The existing management system cannot meet the development of commercialized operation

The management system of sports stadium in China is a kind of management from planning to budget, and from administrative to operative type. Because of the influence of planned economy, both the host and sponsor are lack of realization to the commercialized operation, making that there exist certain problems in the system regulation and equity distribution etc. involved in the commercialized operation of stadium, which buries a hidden danger for the unfair competition, and this is also the direct reason for the low utilization efficiency of labor resources etc. Besides, the operation cost is too high, making the operation efficiency of stadiums not high. Meanwhile, due to different objective conditions and great differences among stadiums, the management systems used in management are also different. Currently in the commercialization process of stadiums, there appears the phenomenon of crossing of more management systems, and even some stadiums adopt the management system of planned economy for management.

The hysteresis of management system increases many obstructive factors for the commercialization of stadiums, mainly reflected in the following: first, there is no clear right, due to vague management right and operation right, there is no high separation between the two, and even they cannot be separated, which will finally causes serious lack of independent operation right of stadium commercialization; second, there is no clear subject, there exist the phenomena such as unclear function, undefined rights and responsibilities and no separation of benefit between the superior competent department and stadiums, so all subjects want to manage the stadiums, which forms repeated management; meanwhile, because of no mutual interference between departments, it is also easy to generate management loophole; third, the subject right is not defined clearly, and the commercialized operation is unclear, there is a great difficulty in the combination of various 
resources, and this is an important reason for the low operation benefit and management efficiency.

(III) Lack of professional talents

Because of the influence of system hysteresis, there will be more personnel in exceeding the staffing in the administrative department of stadiums. Although there are a lot of personnel, the overall quality is low. These overstaffed personnel mainly consist of retired athletes and cadres from sports system etc., and there are very few professional operation and management talents of stadiums. Actually, in the process of commercialized operation, there are very few talents who really master the management and market economy operation law in the stadiums, and this is the main reason to cause that the commercialized operation of stadiums is dominated by extensive management. Because commercialization is seriously obstructed, the economic benefit cannot be fully played, and finally the sustainable development of stadiums will be seriously influenced.

\section{Approach for industrialized operation of stadiums}

(I) Establishing and improving policy and regulations

In order to increase the commercialized operation efficiency of stadiums, it has been urgent to establish regulations and management systems related with the commercialized operation of stadiums, for example, further strengthen supervision and management through the establishment of intellectual property law. Meanwhile, the state and stadium management department should further perfect the laws and regulations for commercialization of stadiums and establish the corresponding legal guarantee system on this basis. Meanwhile, various places can also improve the regional regulations in combination with their own characteristics, and regulate and guide the athletes, clubs and brokers, reasonably coordinate the relation between the equity subjects, mobilize their work initiatives and enthusiasm and make full use of their labor division efficiency. In this way, it is both possible to ensure the high efficiency of commercialized operation of stadiums, and supervise and regulate their commercialized operation.

Provide some preferences for the commercialized operation of stadiums in combination with the actual condition of commercialized operation of stadiums in China, play the government's role in this aspect, so as to further regulate the commercialized development direction and construct a policy service platform related with it on this basis. With the influence of market economy system, it is required to change the functions of government department from micro aspect to macro aspect, the government needs to provide the corresponding funds for commercialized development, and provide orientation in policy, so as to promote the smooth development of stadiums.

(II) Further improve the existing management system

These investing in the stadiums mainly include the state-owned enterprises and institutions, so it is possible to select the sports operation enterprises through bid invitation and bidding for commercialized operation based on this, and the investors mainly guide and supervise the management. In the commercialized operation of stadiums, it is required to continuously improve the existing management system and make it consistent with the requirements for commercialized operation of stadiums. First, the government should treat the commercialization of stadiums in a macro perspective, in the process of commercialized operation of stadium, the government should guide its development direction, create a sound and fair environment for its development and promote the smooth development of its commercialization; second, the enterprises should make clear the development objective of commercialization and continuously enhance the management ability. Therefore, the subject of commercialized operation must be clear, only by clearly defining the responsibilities and rights of the subject will it be able to accelerate the commercialized operation. Meanwhile, the government should correctly position its role, i.e. to carry out macro and guidance for the commercialized operation, create an active development environment for the commercialized operation, reduce government interference and combine with the market law for scientific operation and management to the commercialization of stadiums.

(III) Strengthen the cultivation of comprehensive talents

To promote the sound commercialized operation of stadiums, it is essential to combine with the construction of management talents. Therefore, to retain talents in such a fierce market competition, 
the stadiums must pay attention to the comprehensive cultivation of talents, strengthen the introduction of comprehensive operation and management talents, further strengthen the talent construction, improve the quality of talents overall and continuously improve the operation mechanism. In the process of commercialized operation of stadiums in China, the comprehensive talents can be cultivated through cultivation in universities and the joint cultivation by universities and stadiums, in this way it is possible to improve the talents' relevant knowledge to stadium, and learn a lot of management knowledge, so as to comprehensive the comprehensive quality of talents.

For a long time, the stadiums in China have introduced talents through a single channel, with the influence of commercialization trend, this mode will be broken soon. Meanwhile, it is required to improve the talent structure, actively cultivate and introduce a comprehensive management talents, who should be both familiar with the commercialized operation and master the development law of sports. In addition, it is required to actively encourage the cadres and employees for further study to improve their comprehensive quality, encourage those with sufficient ability to participate in post training to increase their comprehensive knowledge accordingly, so as to achieve the purpose of playing the full ability of talents and solve the quality problems of managers fundamentally.

\section{Conclusion:}

As stated above, it is an inevitable choice to introduce the commercialized operation and management mode into the management of stadium, and also the inevitable result of development of society and economy. However, at present, there still exist some problems in the commercialized operation of stadiums in China. In this paper, the main problems in the commercialized operation of stadiums are elaborated, and corresponding development strategies are proposed aimed at these problems, so as to provide some references for relevant researches.

Acknowledgments

This paper is an education teaching reform program of universities in Guangzhou, topic name: Research and Practice on Cultivating Practical Sports Service Management Talents with University-enterprise Project as the carrier, project number: GZ06029

\section{References}

[1]Cheng Yanfei, Zhang Wenliang, Chen Yuanxin, Preliminary Discussion on Outsourced Service Operation of Large Stadiums [J], Journal of Nanjing Sport Institute (social science edition), 2009 (3)

[2] Wang Bin, Liu Liwei, Bai Jiangtao, Commercialized Operation of Sports Events and its Implementation Strategies [J], China Business \&Trade, 2009 (17).

[3] Kong Pibo, Cui Ruihua, Unbalanced Development of Community Stadium Layout under the Background of Residential Segregation [J], Journal of Shandong Physical Education Institute, 2013 (1).

[4] Chen Yuanxin, Yi Guoqing, Wang Jian, Application of Service Outsourcing in Operation and Management of Stadium [J], Journal of Shanghai University of Sport, 2013 (2)

[5] Yang Xinsheng, Game Analysis on Community Service Evolution of University Stadiums in the Perspective of Paid Operation [J], Sports Science and Technology, 2013 (3)

[6] Yu Lihong, Status of University Sports Resources and Strategy Selection of Configuration Optimization-Take Hangzhou Higher Education Park in Zhejiang as an Example [J], Journal of Beijing Sport University, 2011 (2)

[7] Jia Wenwei, Paid Opening of University Stadiums in Jinzhou-Research on Industrialized Operation Status and Development Strategy, Journal of Harbin Vocational and Technical College, 2012, (2)

[8] Xing Zhouyou, Research on Service Marketing of Large Sports Stadium based on "4R" Theory [J], Journal of Chengdu Sport University, 2012, (6)

[9] Ai Zhenguo, Wang Hao, Shao Changkun, Preliminary Discussion on Operation and Management Mode of Stadiums in Daqing [J], Journal of Chifeng University (natural science edition), 2014 (14). 